$$
\text { "olah-gal" — 2005/7/4 — 13:44 — page 55 — \#1 }
$$

\title{
The "Teaching Mathematics and Computer Science" Journal logo's mathematical background
}

\author{
Róbert Oláh-Gál and Júlia Salamon
}

Abstract. In the present contribution we give an elementary technology for drawing the geodesics, paracycles and hypercycles on the pseudosphere.

Key words and phrases: Bolyai-Lobachevsky plane geometry, pseudosphere, Poincaré half-plane logical model, geodesics, paracycles and hypercycles.

ZDM Subject Classification: G05, G45, P05, G55.

\section{Introduction}

In the middle of the small saloon at the Bolyai Museum in Marosvásárhely there is a black painted, lumber turned pseudosphere model, and on it is drawn a triangle with the scope and legend to exemplify the non-Euclidean geometry (see pictures 1 and 2). It may be for over 40 years that it has been standing in the middle of saloon.

This is what is shown on television, and can be found on many postcards too. Many times when the materialization of the Bolyai-Lobachevsky geometry on the pseudosphere is exemplified, a triangle is drawn on its surface, and it is written aside, that the summation of the triangle's angles measure less then $180^{\circ}$. Unfortunately these figures are mathematically wrong, because in this surface instead of lines we have geodesics, and in those figures they no geodesics have drawn. The geodesics in the Bolyai Museum are wrong too, the lower geodesic

Copyright (C) 2005 by University of Debrecen 


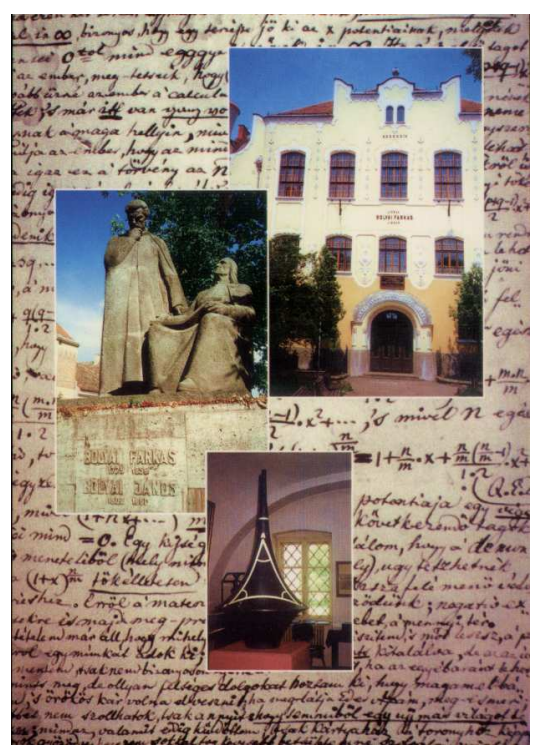

Figure 1. Postcard at Bolyai Museum

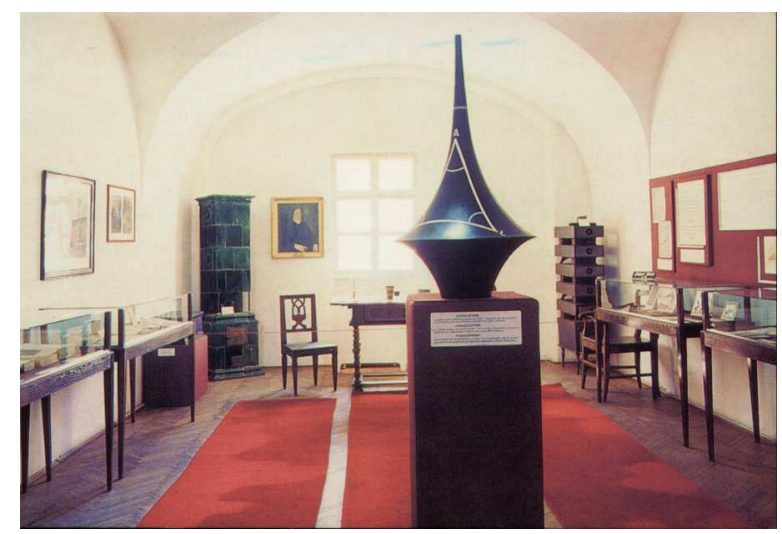

Figure 2. Pseudosphere model in the Bolyai Museum

is also plane curve, (so the intersection of a pseudosphere and a plane, extremely wrong!), and the climbings of the other two geodesics are also wrong.

If we draw a triangle in a surface, and we analyze the summation of angles as an inner and elementary geometrical fact, then the triangle has to have geodesics as components. If we don't think in this way, we could simply draw on the plane 


$$
\text { "olah-gal" — 2005/7/4 — 13:44 — page } 57 \text { — \#3 }
$$

one triangle with arc components, and even then we could determine that the triangle's angle measure is less then $\pi$. Consequently we have to work strict with notions and we have to pay attention to the fundamental properties of the inner geometry.

In case of the pseudosphere this is extremely important because the pseudosphere belongs to those scant surfaces which have a constant negative curvature. On a small domain (on a very small part) it prevails the plane geometry. But attention, it would be a mistake to say, that the Bolyai-Lobachevsky geometry is materialized on it, as well as to declare, that it serves as a model for the BolyaiLobachevsky geometry. This surface is not complete, and the whole area is finite, too.

Even his lines, the geodesics are finite, they proceed from singular circle and they coil on the pseudosphere until they reach one particular parallel circle. We have calculated and illustrated these geodesics.

We can draw a correct conclusion, by saying, that the BolyaiLobachevsky plane geometry is materialized in every point of a very small neighborhood on the pseudosphere!

It's hard to draw the geodesics only with the classic representative of the geometry design. Back in 1993 with the guidance of Dr. Szabó József at the University of Debrecen, Institute of Informatics, we had these geodesics drawn on drawing-table by the use of computer graphics. The deep epistemology and mathematical history background of this simple drawing made the "Teaching Mathematics and Computer Science" journal's editorial board choose this drawing as a logo for there journal.

We can assure about the correctness of the geodesic's drawings by the evocation of Clairaut's theorem. According to Clairaut's theorem the product of the radius of parallel circles and the cosine of angle between geodesics and parallel circles is constant in case of surfaces of revolution. Based on this theorem every meridian curve is geodesic, since the meridian curves are perpendicular to the parallel circles, so this way the cosine of angle is zero and the constant in the theorem is zero too and independent of the radius. It's easy to deduce that in case of the cylinder the parallel circles are geodesics. So in the case of the pseudosphere the meridian curves are geodesics, moreover even those curves that satisfy Clairaut's theorem are geodesics too. Namely they wrap gradually around the pseudosphere until they reach oscillatory one parallel circle, because then $\cos 0=1$ so the prescribed constant is the radius of the reached parallel circle. 


$$
\text { "olah-gal" — 2005/7/4 — 13:44 — page 58 — \#4 }
$$

The fact is that the Bolyai-Lobachevsky plane geometry materializes only in a very small neighborhood on the pseudosphere, as same as the Euclidean geometry materializes on two spliced conical surfaces. The Euclidean geometry is also materialized in small on the whole conical surface or cylindrical surface, but even these are better models than the pseudosphere because they have an infinite area. And the pseudosphere has a finite area! In spite of this it elongates into infinity, the whole area is $4 \pi$, if the circle is unit radius. So similar to the sphere whose whole area is $4 \pi$, if the radius of sphere is 1 . Unfortunately, the Bolyai-Lobachevsky plane geometry doesn't have a natural model, as does the Euclidean geometry, namely the ordinary plane surface. (As far as we know the Bolyai-Lobachevsky plane geometry has a natural model only in 6 dimensional spaces; it has many logical models: the Poincaré half-plane model, the CayleyKlein, Klein-Poincaré, Némethi András, Kőnig Gyula, Nicolae Mihăilenu models, etc.)

Such an important visual aid of a scientific weight can not be considered to be wrong.

The curiosity of the scientific disquisition is that only now do we have at our disposition a very old book, which offers a simple and original method of drawing the geodesics. But with the help of this we can draw easily not only the geodesics on the pseudosphere, but even the para- and hypercycles. And this is not included in a single book, journal or website known by us. Well one of our aims within this paper was to present the paracycles on the pseudosphere. It's known that in Bolyai-Lobachevsky geometry every paracycle is congruent, is removable in it self, and also that it has the same property correlated to every point, a totally regulate curve. This means that on the whole hyperbolical plane we could create line ruler and paracycle ruler. But in this figure it will be visible that the pseudosphere is only a local model, because the paracycles on the pseudosphere will not be congruent!

For drawing geodesics, paracycles and hypercycles the algorithm is: In every case, first we represent the famed lines in the Poincaré half-plane model, then we transform these lines inside of a circle, and from here we project them on the pseudosphere.

The steps of drawing the geodesics: The Poincare half-plane model is known, the $e$ line is given which divides the plane into two parts, and for the hyperbolic plane we consider one of the half-plane's points, in such a way that the points of the given line are the infinite distant points; thus, those don't belong to the hyperbolic half-plan's points. For the simplicity without loss of generality we can 


$$
\text { "olah-gal" — 2005/7/4 — 13:44 — page } 59 \text { — \#5 }
$$

consider the $\mathbb{R}^{2}$ (xoy, $y>0$ ) plane. The half-plane model is easy to be associated with the computer's screen. We can one-to-one associate the hyperbolic plane with the half-plane, if the distance between two points is given with $d(x, y)=$ $k \cdot \log (x y v u)$ coordinates, where $(x y v u)$ is the cross ratio. The lines (geodesics) are given by half-circles, with origin on the $e$ boundary line. So we have to transform these on the pseudosphere. The essential of all of this is the fan method, when in one rectangle we obtain one circle (fan), in such a way that we shrink one side of the rectangle into one point.

The algorithm of drawing the geodesics (fan method): Drawing the halfcircle with $r$ radius (geodesic) on the Poincaré half-plane, the coordinates of the half-plane are in $x, y$ array, where in $o$ array is the argument.

$$
\begin{aligned}
& o=0: 0.01: p i \text {; } \\
& n=\text { length }(o) \text {; } \\
& \text { for } i=1: n \\
& x(i)=r * \cos (o(i)) \text {; } \\
& \text { end; } \\
& y(i)=r * \sin (o(i)) \text {; }
\end{aligned}
$$

We take the band between the lines $x=\pi$ and $x=-\pi$. If the radius of the half-circle's is bigger then $\pi$, then we project the half-circle symmetrically back regarding the lines $x=\pi$ and $x=-\pi$, until every part of the half-circle will be in the $[-\pi, \pi]$ band.

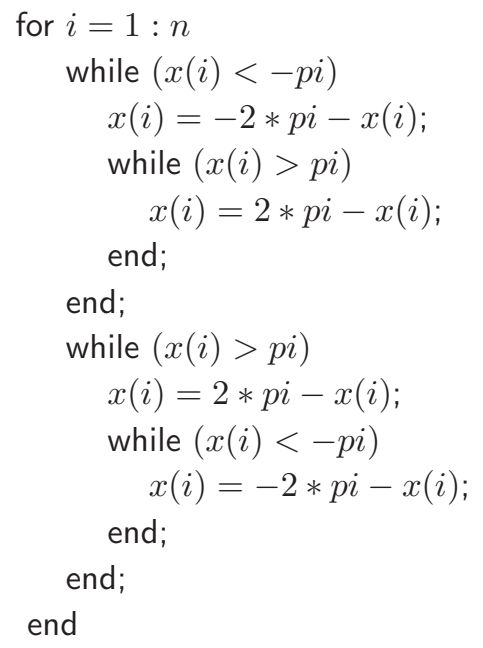

The next step is the transformation of the obtained curve inside of one circle with radius $r+1$. 

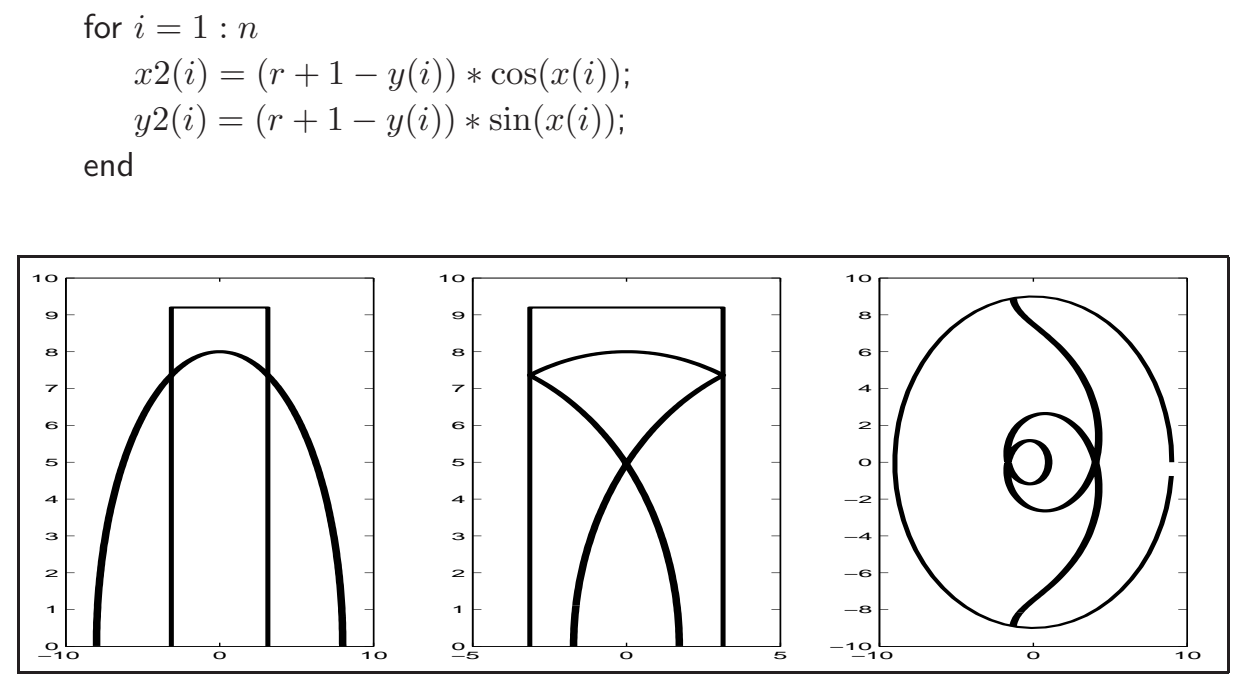

Figure 3. Geodesics in the Poincaré half-plane, in the $[-\mathrm{Pi}, \mathrm{Pi}]$ band, and in the circle

We only have to project the points of the curve inside the circle into the pseudosphere (see Figure 4), knowing the formula of the pseudosphere

$$
\left\{\begin{array}{l}
x=r u \cos (v) \\
y=r u \sin (v) \\
z=r \log \frac{r+\sqrt{r^{2}-\left(y^{2}+x^{2}\right)}}{\sqrt{y^{2}+x^{2}}}-\sqrt{r^{2}-\left(y^{2}+x^{2}\right)}
\end{array}\right.
$$

where $u$ is the height (altitude) of the parallel circles, and $v$ is the angle inside of these parallel circles which value is in $[-\pi, \pi]$ interval.

Drawing a geodetical triangle with this method, doesn't mean any difficulty. We take an intersection by 3 lines (half-circles) on the Poincaré half-plane model, we wedge into $2 \pi$ height band, and then we transform each one into one circle, where we project them into the pseudosphere (see Figure 5).

The drawing of paracycles follows the algorithm of geodesics drawings. In the Poincaré half-plane model the paracycles are the circles that contact the boundary line (we consider those specific circles which have the center on the line that is perpendicular on the boundary line in the origin), respectively the parallel lines with the boundary line. The drawing of ulterior paracycles is not a problem because these are the parallel circles on the pseudosphere, while in case of the 


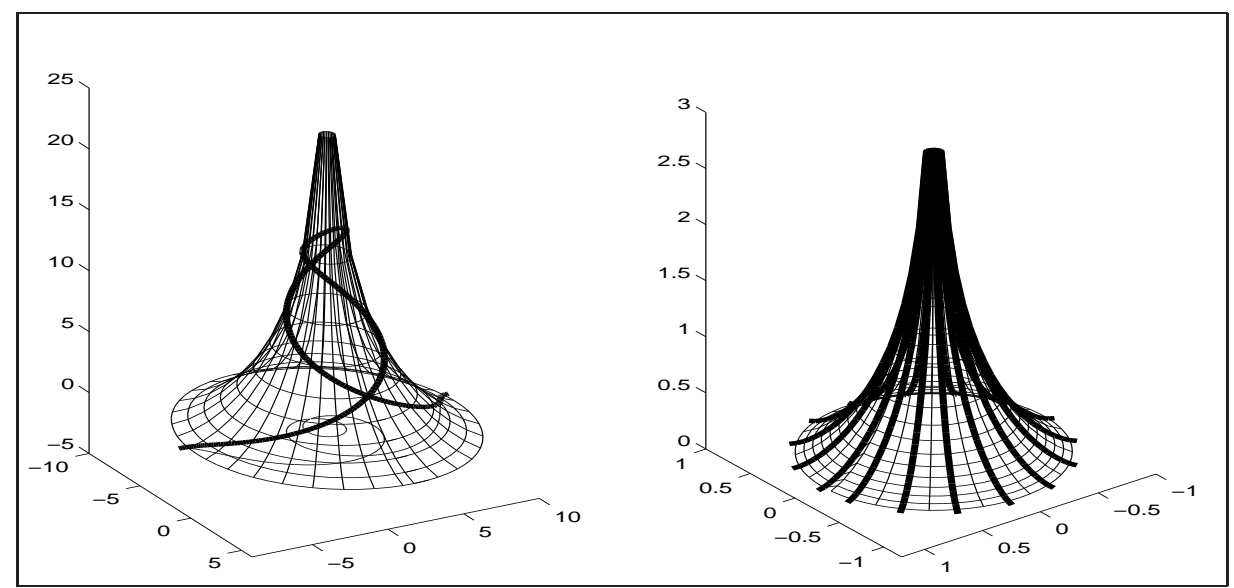

Figure 4. Geodesic on the pseudosphere

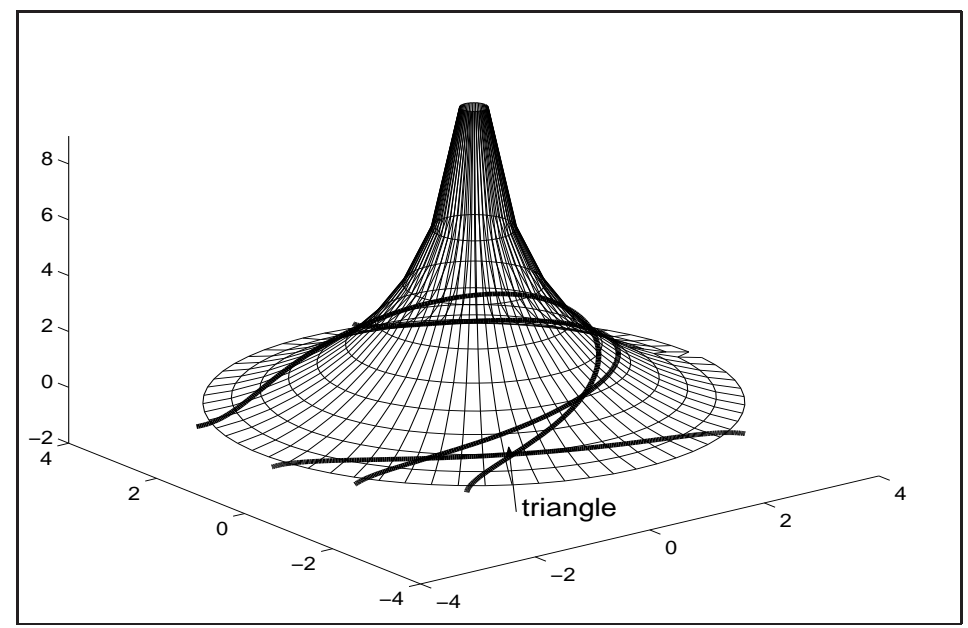

Figure 5. Triangle on the pseudosphere

other paracycles, we proceed the same way as we described above (see Figures $6,7)$.

The algorithm of the drawing of hypercycles. To draw a hypercycle first we have to give the geometric locus of the points which are at a given distance to the line (half-circle) in the Poincaré half-plane model. The procedure is the same as in case of Euclidean geometry. We draw in given line's points one perpendicular 


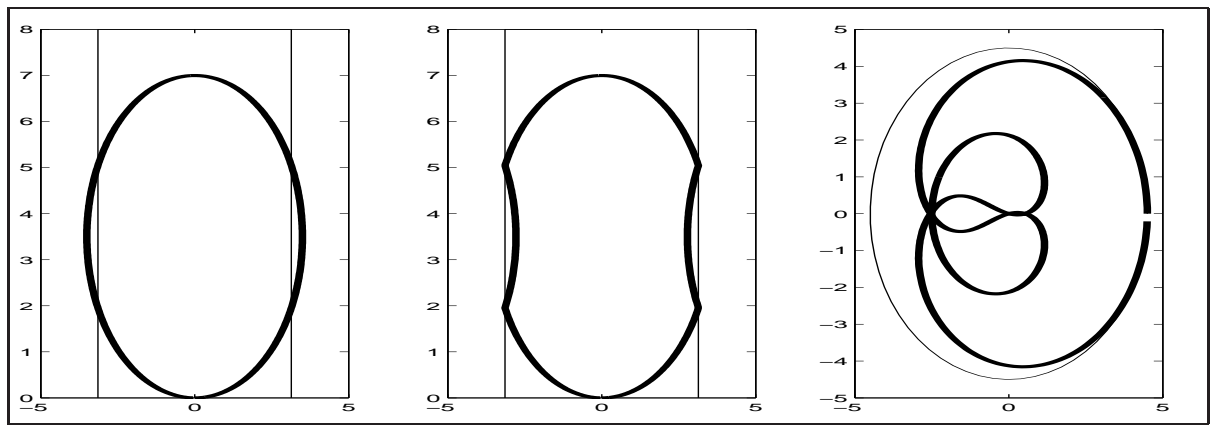

Figure 6. Paracycle in the Poincaré half-plane

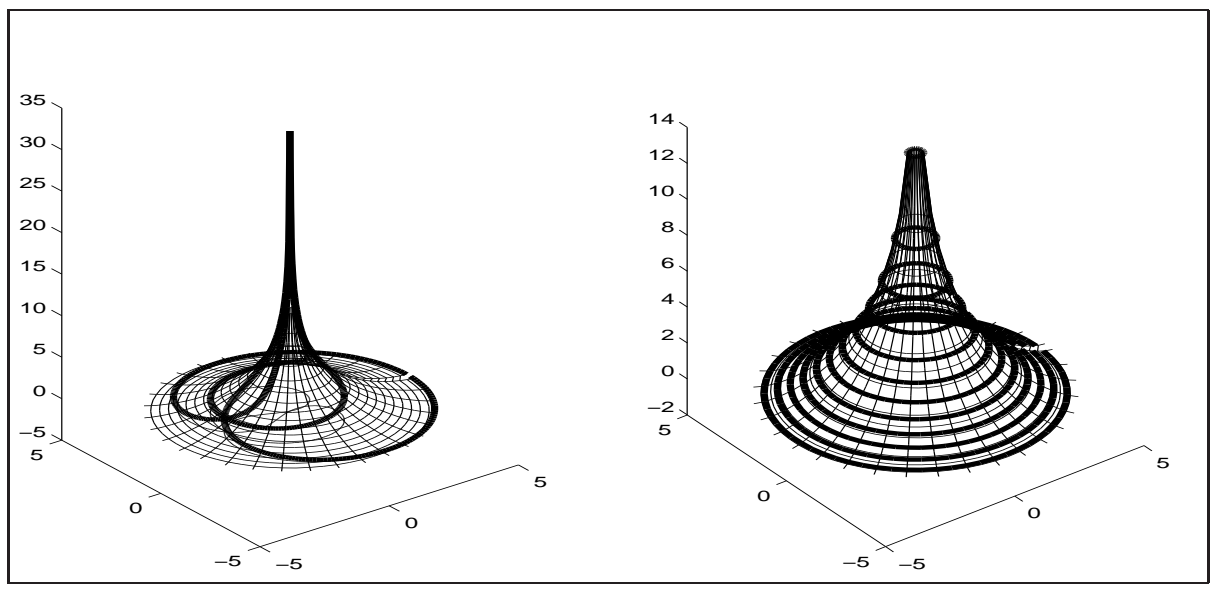

Figure \%. Paracycle on the pseudosphere

line, where we measure the given distance. The points obtained in this way are in the parallel line at a given distance at the line. In the Poincaré half-plane it will be a little bit different, here the line is a half-circle, the lines which are perpendicular at the line, are perpendicular intersected half-circles of the halfcircle (the tangent lines are perpendicular in the intersection). The radii and the centers of these half-circles are given by the formulas

$$
\begin{aligned}
r a d & =r \cdot \tan \left(o-\frac{3 \pi}{2}\right) \\
x c & =-\sqrt{r^{2}+r a d^{2}} .
\end{aligned}
$$


On these half-circles we have to measure the given distance which in this case is defined by

$$
D(A, B)=k \cdot \log (A B V U)
$$

metrics, where $k$ is one arbitrary positive constant, and with $(A B V U)$ we sign the cross ratio

$$
(A B V U)=\frac{A V}{B V} \cdot \frac{B U}{A U}
$$

where $A, B$ are two points on the half-circle (line) and $V, U$ are the intersection points of the half-circles and the boundary line. More precisely:

$$
d\left(\left(x_{1}, y_{1}\right),\left(x_{2}, y_{2}\right)\right)=\frac{1}{2} \log \frac{\left(\left(x c-r a d-x_{1}\right)^{2}+y_{1}^{2}\right) \cdot\left(\left(x c+r a d-x_{2}\right)^{2}+y_{2}^{2}\right)}{\left(\left(x c+r a d-x_{1}\right)^{2}+y_{1}^{2}\right) \cdot\left(\left(x c-r a d-x_{2}\right)^{2}+y_{2}^{2}\right)}
$$

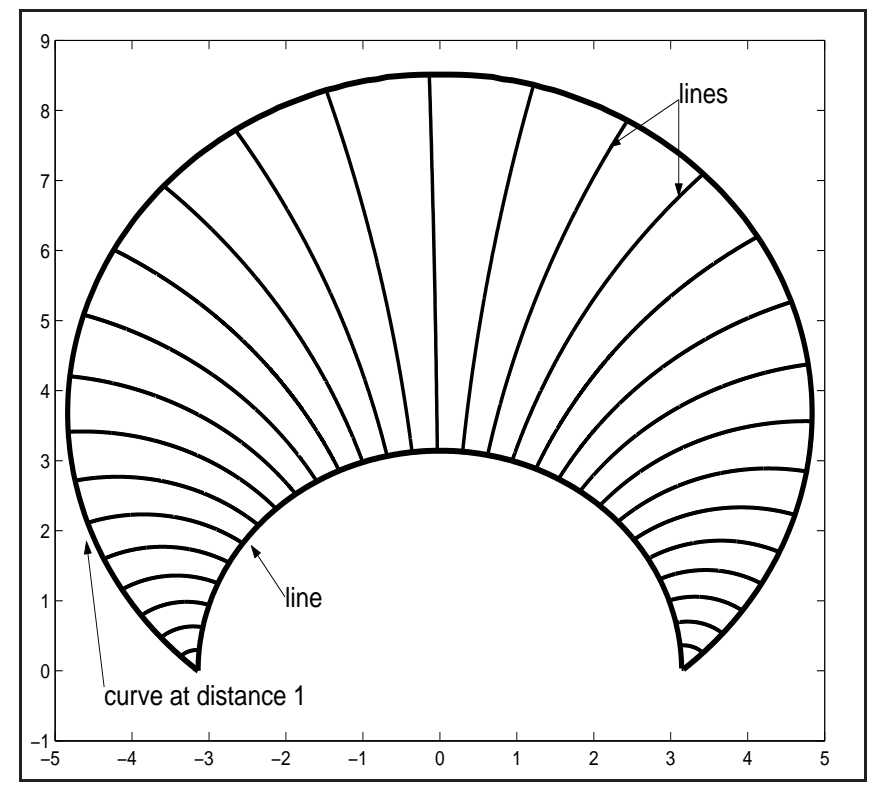

Figure 8. Hypercycle in the Poincaré half-plane

We project the points obtained in this way, which are on a curve (not line (half-circle)) (Figure 8) into the pseudosphere by the described method (Figure $9)$. 


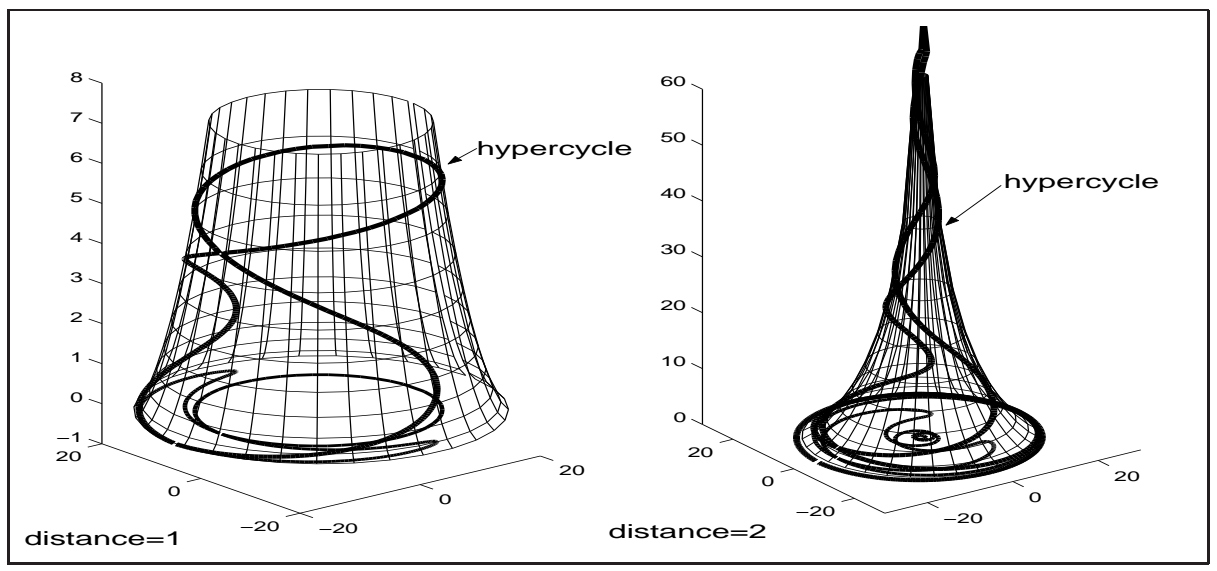

Figure 9. Hypercycle on the pseudosphere

\section{Conclusions}

From a didactical aspect it is very hard to exemplify a geometrical fact with mathematically correct figures. Subsequently it is very hard to change one wrongly evolved geometry image. One typical example of this is the illustration of an ellipse as a plum core, albeit the ellipse is an analytical curve, so it doesn't have angle points.

Maybe the idea of editors of "Teaching Mathematics and Computer Science" to choose as the review's logo a geodetical triangle design on the pseudosphere will not be useless.

\section{Acknowledgments}

The work of the authors was supported by the Research Programs Institute of Foundation Sapientia, Grant Nr. 1898/19.12.2003.

\section{References}

[1] F. Schilling, Die Pseudosphäre und die nichteuklidische Geometrie, Teubner, Leipzig und Berlin, 1935, 215.

[2] Tibor Weszely, A Bolyai-Lobacsevszkij geometria modelljei, Dacia Könyvkiadó, 1975. 


$$
\text { "olah-gal" — 2005/7/4 — 13:44 — page 65 — \#11 }
$$

[3] Róbert Oláh-Gál, Despre geodezicele pseudosferei (On the geodesics of pseudospheres), (Romanian) Proceedings of symposium in geometry (Cluj-Napoca and Tîrgu Mureş, 1992), 133-136, preprint, 93-2, Babes-Bolyai Univ., Cluj-Napoca, 1993.

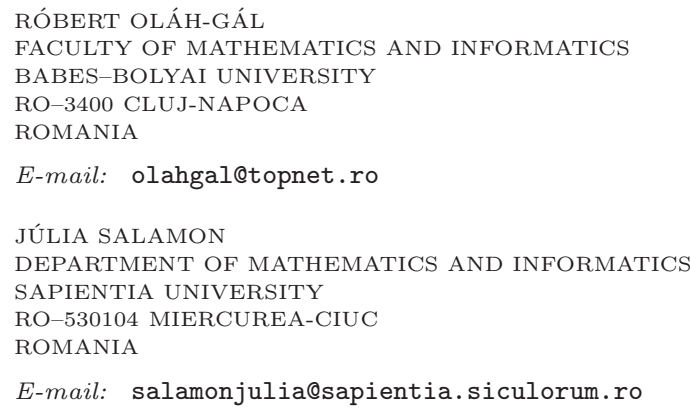

E-mail: salamonjulia@sapientia.siculorum.ro

(Received November, 2004) 